Volume 8, No.2, March - April 2019

International Journal of Information Systems and Computer Sciences

Available Online at http://warse.org/IJISCS/static/pdf/file/ijiscs34822019.pdf

https://doi.org/10.30534/ijiscs/2019/34822019

\title{
Hyper Spectral Prevalent News Customization System With Sentimental Analysis
}

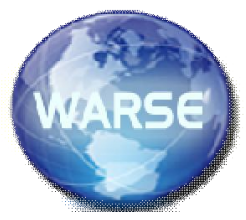

ABSTRACT

Broad communications sources, specifically the news media, have traditionally informed us of daily events. News customization play an important role in news media. Extracting knowledge of certain incident from different online portals into a single online portal. The news media introduces expertly verified events or occasions, while web based life displays the interests of the group of onlookers in these regions. Users can see the multiple news website news in a single portal. The online news media shows the verified news only. Repetition of news is avoided. Users can share the valuable information from online news media to online social media. Online user generated reviews become an inevitable part of decision making process. Natural language processing technologies is used for online reviews. Analyzing the overall sentiment expressed in a whole piece of text or in the form of like or dislike.

Key words :Broad communications, Decision making, Natural Language processing, News customization

\section{INTRODUCTION}

The mining of profitable data from online resources has become a noticeable area in information technology. In modern times social media services provide an enormous amount of data. Here we introduce news media which provide valuable information from different online portals. Identifying topics from different social and news media sources is the application of topic modeling. Many methods have been proposed[1],[2],[3].The news media displays expertly verified events or occasions Repetition of news is avoided. In case of social media services such as face book, twitter users can generate fake news to the public. The news media services provides only the valuable amount of information and the fake news are not permitted. A single online portal consists of news from different news media. News media sources are published by professional journalist. The news in news media are verified. Social media presents only the interest of the public.

In social media services the users generate repetitive news whether it is fake or not and non journalist users publish not verified content and express their interest in certain news events. User generated reviews play an important role in online news media's. Based on online user generated reviews and ratings we can analyze the sentiments. Users can add their own opinion and analyze each topic based on the user generated reviews.
Users can add comments as a piece of text and like or dislike the topics. Based on online reviews users can analyze each topics sentiments and can arrive at a common opinion. News topics are ranked based on the media focus, user attraction, user interaction.

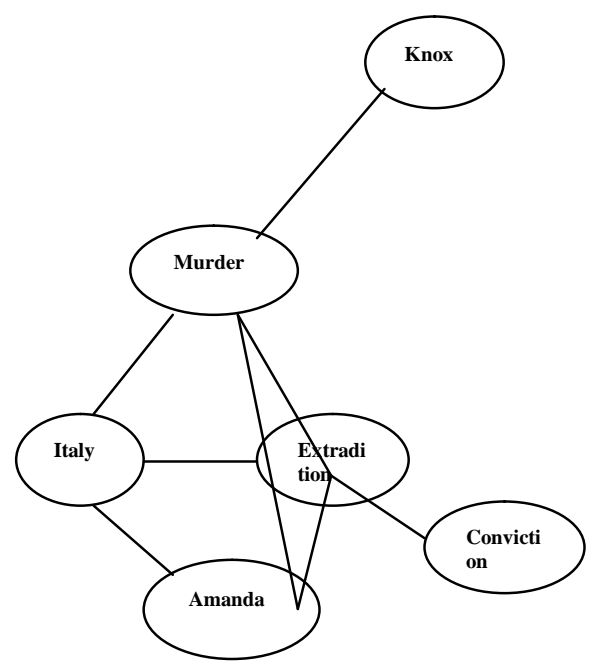

Figure 1:Topic Relationship

Topics related to Amanda Knox murder trial is represented by the terms Amanda, murder, Knox etc. From this it shows the relationship between the topics. The similar topics are connected together. The news which are under the same category are connected.

News media provide an enormous amount of data. The data's from news media are considerably valuable. The news from news media's are published by professional journalist. The news from different online portals can be available in a single online portal. It is a time consuming technique. Through single portal users can access different online news channels. News from news media's are ranked based on the media focus, user attraction and user interaction. The users can provide online reviews as comments. Repetition of news is avoided. Online reviews play an important role in news media. The reviews can be postive,negative or neutral[4].The users can analyze about a topic through reviews. Sentiment analysis can be done through a single piece of text like or dislike.

Classifying a review document into positive or negative sentiment[5],[6].Here the news from different online news portal can be available in a single online portal. 


\section{RELATED WORK}

The principle research are in this paper includes subject identification, ranking topics, network analysis, keyword extraction, graph clustering etc.

Research play an important role in topic identification. Two methods for detecting topics are LDA and PLSA.LDA model can be applied to different tasks including subject identification. PLSA which can be applied to topic modeling. In these techniques temporal information is lost, which is paramount in identifying important topics and is an important characteristics of social media data. Another major concept in this paper is topic ranking. The proposed method that takes into account the users interest in a topic by estimating the amount of times they read stories related to that topic. Ranking the news topics based on this. the energy of a topic increases only when it becomes popular[7]. Network analysis defines the user attraction. When a user comment or reacted to a topic it describes the user attraction to the topic. It shows the users interest towards the topic. Soci rank which identifies the news topics based on user attraction, user interaction and media focus. News topics are ranked based on the analysis of user interaction.

Effective algorithms are used for keyword extraction. Many supervised unsupervised methods are used for keyword extraction[9]. Supervised methods make use of training datasets that have already been classified. Among the unsupervised methods, there are those that employ statistical measures. Other unsupervised approaches are graph-based, where a text is converted into a graph whose nodes represent text units .

The main purpose of graph clustering also proposed a method that clusters a co-occurrence graph based on a graph measure known as transitivity. The basic idea of Graph clustering is transitivity. The core idea behind Newman clustering is the number of shortest paths between pairs of nodes. The users can post their own tweets. Sentiments can be analyzed through user generated reviews. The reviews can be either positive or negative. Bullying comments can be encrypted automatically. Sentiments can be analyzed from a piece of text also. Opinions can be analyzed at word or phrase levels.

A sentiment s, or opinion, refers to the semantic orientation and strength of satisfaction on a reviewed entity .Positive semantic orientation indicates "good", while negative semantic orientation indicates "bad". Overall rating indicates the strength of sentiment on a reviewed document. semantic aspect detection aims at detecting the hidden semantic aspects. aspect level sentiment identification aims to identify fine grained semantic sentiments. The fine grained sentiments can be either positive or negative.

\section{PROPOSED SYSTEM}

Soci-rank framework which shows the prevalent news topics in news media and show it's sentimental analysis. News topics are ranked based on the media focus, user attraction, User interaction. News from different online portals are customized into a single online portal. All the news from different online portals are categorized into sports, education health etc. The users can post their own posts from news media. The news from the users are verified. The news from social media can be generated by journalist or non journalist In this paper all the news are verified by the admin. So there is no chance of fake news The Sentimental analysis which shows the popularity of the news topics. Users can analyze the news topics based on the sentimental analysis and the users can arrive at a conclusion. All the data's are stored in the database.

\subsection{Soci Rank Framework}

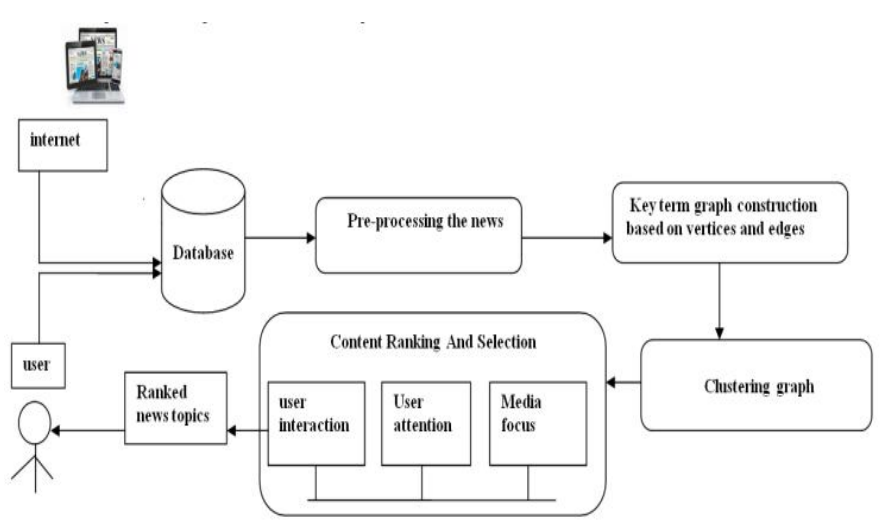

Figure 2:Soci Rank Framework

\section{A.Preprocessing Stage}

In the preprocessing stage all the news articles, tweets fall inside the database. In case of news term extraction the keywords are extracted from database[8].Removes all the bullying comments by analysing.

\section{B .Key Term Graph Construction}

A graph $\mathrm{G}$ is constructed whose nodes represent the news topics in news media and social media. Edges shows the relationship between nodes.

\section{Selection and Ranking}

News topics are ranked based on the media focus, user attraction and user interaction. The popularity of the news can be analyzed through this. The users can analyze the topic based on the sentiments. The users can like or dislike the news topic or they can provide comments as a piece of text. The bullying comments can be encrypted

\subsection{News Customization}

News from different online portals like Hindu, Indian Express etc are available in a single online portal. News topics are categorized. The repetition of news is avoided. The news topics are verified because news customization is taking place from different online portals. The news from 
different news media's are generated by professional journalist. In social media most of the news are unverified. All the news are generated by non journalist. Here news from different online portals are customized into a single portal. The users can easily chose the news website from a single portal. The users can post their own tweets to the news media only when admin is approved. If users post any fake news the admin will automatically reject the user.

Text mining algorithm is used for news extraction. Text mining is defined as the extraction of useful content from the natural language text. The algorithm which removes all the unwanted data's .Text mining which is similar to data mining. Information extraction play an important role in news customization. Information extraction is the task of automatically extracting structured information.

\subsection{Sentimental Analysis}

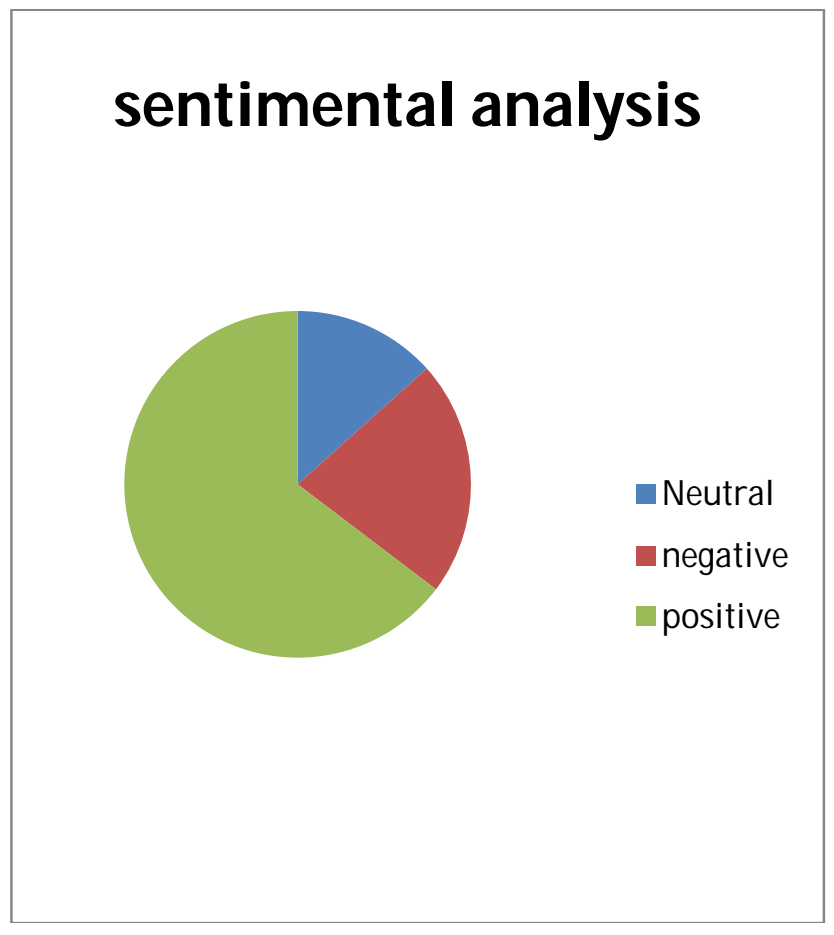

Figure 3: Analyzing sentiments

Sentiments or opinion can be either neutral, negative, or positive. positive semantics indicates good, while negative semantics indicates bad neutral means it either belongs to good or bad. the above graph is just an example graph shows the sentimental analysis of different posts from different users. The opinion of topics varies according to the users comments. This graph shows that $46.8 \%$ of tweets are neutral,13.5\% tweets are negative. $39.7 \%$ of tweets are positive.

Opinion mining refers to natural language processing. Basic fact in opinion mining or sentimental analysis is classifying the polarity of the given textual document. Encryption algorithm is used for sentimental analysis. Transforming bullying comments into encrypted text.

\section{RESULT}
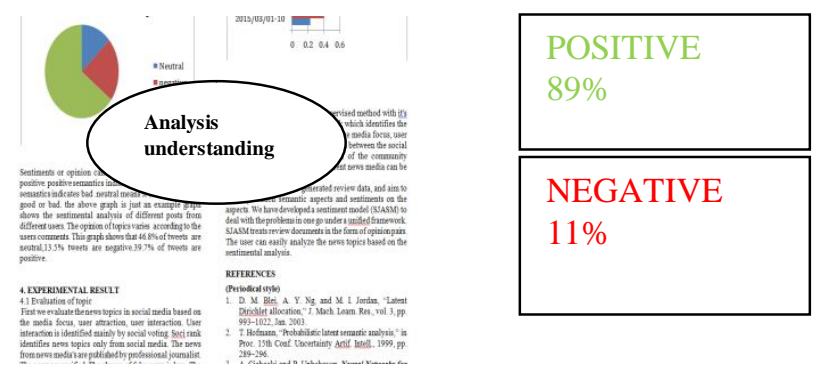

Figure 4:Analysis Understanding

First we evaluate the news topics in social media based on the media focus, user attraction, user interaction. User interaction is identified mainly by social voting. Soci rank identifies news topics only from social media. The news from news media's are published by professional journalist. The news are verified. The chance of fake news is less. The news from different portals can be available in a single portal.

We evaluate the ranking of news topics using the soci rank and media focus[10].

\section{CONCLUSION}

In this paper, we proposed an unsupervised method with its sentimental analysis is used. Soci rank which identifies the news topics in social media based on the media focus, user attraction, user interaction. Interaction between the social media which indicates the strength of the community discussing over it. News from different news media can be available in a single portal. We focus on online usergenerated review data, and aim to identify hidden semantic aspects and sentiments on the aspects. The user can easily analyze the news topics based on the sentimental analysis.

\section{REFERENCES}

1. D. M. Blei, A. Y. Ng, and M. I. Jordan, "Latent Dirichlet allocation,” J. Mach. Learn. Res., vol. 3, pp. 993-1022, Jan. 2003.

2. T. Hofmann, "Probabilistic latent semantic analysis," in Proc. 15th Conf. Uncertainty Artif. Intell., 1999, pp. 289-296.

3. A. Cichocki and R. Unbehaven. Neural Networks for Optimization and Signal Processing, 1st ed. Chichester, U.K.: Wiley, 1993, ch. 2, pp. 45-47.

4. P. D. Turney and M. L. Littman, "Measuring praise and criticism: Inference of semantic orientation from association," ACM Trans. Inf. Syst., vol. 21, no. 4, pp. 315-346, Oct. 2003.

https://doi.org/10.1145/944012.944013 
5. B. Pang, L. Lee, and S. Vaithyanathan, "Thumbs up?: sentiment classification using machine learning techniques," in Proceedings of the ACL-02 conference on Empirical methods in natural language processing Volume 10, ser. EMNLP'02. Stroudsburg, PA, USA: Association for Computational Linguistics, 2002, pp. 79-86 https://doi.org/10.3115/1118693.1118704

6. V. Ng, S. Dasgupta, and S. M. N. Arifin, "Examining the role of linguistic knowledge sources in the automatic identification and classification of reviews," in Proceedings of the COLING/ACL on Main Conference Poster Sessions, ser. COLING-ACL '06. Stroudsburg, PA, USA: Association for Computational Linguistics, 2006, pp. 611-618.

7. C. C. Chen, Y.-T. Chen, Y. Sun, and M. C. Chen, "Life cycle modeling of news events using aging theory," in Machine Learning: ECML 2003. Heidelberg, Germany: Springer Berlin Heidelberg, 2003, pp. 47-59. https://doi.org/10.1007/978-3-540-39857-8_7

8. R. Mihalcea and P. Tarau, "TextRank: Bringing order into texts," in Proc. EMNLP, vol. 4. Barcelona, Spain, 2004.

9. K. Kireyev, "Semantic-based estimation of term informativeness," in Proc. Human Language Technol. Annu. Conf. North Amer. Chapter Assoc. Comput. Linguist., 2009, pp. 530-538. https://doi.org/10.3115/1620754.1620831

10. Google News. [Online]. Available: http://news.google.com, accessed Feb. 2014 